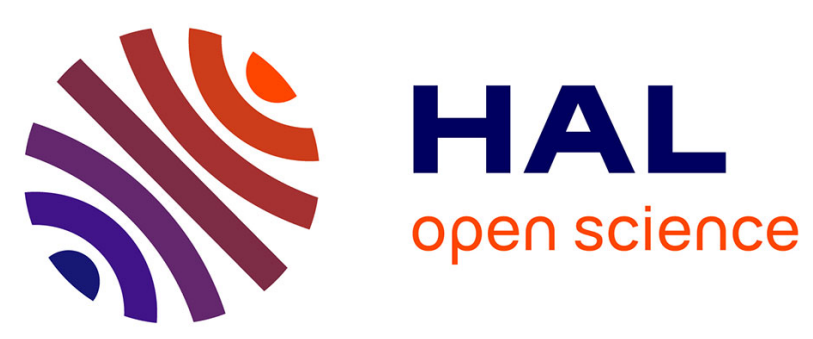

\title{
Optical-Flow Based Strategies for Landing VTOL UAVs in Cluttered Environments
}

Lorenzo Rosa, Tarek Hamel, Robert Mahony, Claude Samson

\section{To cite this version:}

Lorenzo Rosa, Tarek Hamel, Robert Mahony, Claude Samson. Optical-Flow Based Strategies for Landing VTOL UAVs in Cluttered Environments. 19th World Congress. The International Federation of Automatic Control., Aug 2014, Cape Town, South Africa. pp.3176 - 3183, 10.3182/20140824-6ZA-1003.01616 . hal-01342055v2

\section{HAL Id: hal-01342055 \\ https://hal.science/hal-01342055v2}

Submitted on 11 Oct 2016

HAL is a multi-disciplinary open access archive for the deposit and dissemination of scientific research documents, whether they are published or not. The documents may come from teaching and research institutions in France or abroad, or from public or private research centers.
L'archive ouverte pluridisciplinaire HAL, est destinée au dépôt et à la diffusion de documents scientifiques de niveau recherche, publiés ou non, émanant des établissements d'enseignement et de recherche français ou étrangers, des laboratoires publics ou privés. 


\title{
Optical-Flow Based Strategies for Landing VTOL UAVs in Cluttered Environments
}

\author{
Lorenzo Rosa* Tarek Hamel ?? Robert Mahony ** \\ Claude Samson?? \\ ${ }^{*}$ L. Rosa is with the Dipartimento di Ingegneria Informatica, \\ Automatica e Gestionale, Sapienza University of Rome, Italy. \\ rosa@diag.uniroma1.it \\ ${ }^{* *}$ R. Mahony is with the Research school of Engineering, Australian \\ National University, Australia.
}

\begin{abstract}
This paper considers the question of landing an Unmanned Aerial Vehicles (UAV) using a single monocular camera as the primary exteroreceptive sensing modality. The proposed control law is based on tracking a single point feature, representing the desired landing point on a ground plane, along with optical flow computed over the full image. The bearing of the desired landing point is used as a driving term to force convergence, while the optical flow is used to provide a damping force that guarantees both obstacle avoidance as well as damping the convergence of the vehicle to the ground plane ensuring a soft touchdown. A detailed analysis of the system closed-loop dynamics is undertaken and the response of the system is verified in simulation.
\end{abstract}

Keywords: Aerial Robotics, UAV, VTOL, visual servo control, optical flow, landing.

\section{INTRODUCTION}

Autonomous aerial vehicles (UAVs) are a transformative technology in modern society, providing unparalleled capability to undertake difficult, dangerous and dull surveillance and monitoring tasks crucial to maintenance of infrastructure, agricultural operations and disaster recovery, to name just a handful of application domains. To perform a fully autonomous flight, an UAV requires the ability to take-off, navigate to, and accomplish a given task, and finally return to base and land. Many tasks require the vehicle to land, or at least approach and touch, its destination, and complex or long-lasting missions might require intermediate landing and taking-off operations (e.g. battery/fuel refill). Such manoeuvres generally need to be undertaken in unsurveyed, cluttered environments, and often where GPS signals are unreliable or unavailable. This is especially the case for Vertical Take-Off and Landing (VTOL) vehicles, such as helicopters and multirotor systems, that are likely to be used in tandem with ground based vehicles and other technologies, as in Michael et al. (2012), in extended disaster recovery or other types of missions. Classical landing control strategies require estimation of the full state of the vehicle, along with a model of the landing environment (see for example Flores and Milam (2006); Geyer and Johnson (2006); Meister et al. (2009); Andert et al. (2011)). If only a camera is available for state estimation then the vehicle pose must be estimated by using visual data (Lee et al., 2012; Davison et al., 2011; Kaiser et al., 2010; Courbon et al., 2010; Milford et al., 2011; Blösch et al., 2010) An alternative approach is to use the natural properties of optical flow to provide a strong visual cue for obstacle avoidance and landing regulation (Srinivasan et al., 2000; Koenderink and van Doorn, 1987; Hérissé et al., 2008; Chahl et al., 2004; Srinivasan et al., 2000; Ruffier and Franceschini, 2004). Optical flow is also a powerful cue for terrain following (Humbert et al., 2005; Ruffier and Franceschini, 2005) and obstacle avoidance Geyer and Johnson (2006); Green and Oh (2008); Beyeler et al. (2009). A good review of prior work in robotics applications that exploit optical flow is given by McCarthy et al. (2012). A key advantage of control strategies that are based on optical flow is that they also also deal well with dynamic environments such as a moving landing surface (Hérissé et al., 2010, 2012) in contrast to approaches that require modeling of the environmental motion (Marconi et al., 2002). A disadvantage of control algorithms based on optical flow and visual data in general is the complexity of providing rigourous stability analysis of the closed loop system, especially in the case where the system has non-trivial dynamics, as is the case with flying vehicles.

In this paper, we propose a landing control algorithm for a VTOL UAV based on visual data obtained from a monocular camera coupled with the usual suite of sensors present in a standard Inertial Measurement Unit (IMU) that is common on all modern UAVs. We provide a detailed analysis of the closed-loop response of the system and prove asymptotic stability of the pose to the desired landing point, corresponding to a smooth touch down. We first consider the case where there are no obstacles and propose a reformulation of the controller formerly developed by Hérissé et al. $(2010,2012)$ to include a target landing point. The proposed control consists of a driving term that acts as a constant force driving the 
vehicle in the direction of the observed target point, based on the bearing measurement of the target, coupled with a damping proportional to optical flow measured over the whole ground plane. The natural tradeoff between velocity and distance inherent in optical flow ensures a stable soft touchdown of the closed-loop system. We then consider obstacles present in the flying space by including additional damping terms associated with divergence of the optical flow derived from the obstacle. To simplify the mathematical formulation of the problem, the ground plane is assumed to be flat and obstacles are modeled as spheres in space. The goal of this paper is to provide a detailed theoretic analysis of the system response rather than tackle some of the practical issues of implementation. To this end the visual target observation and optical flow measurements are abstracted and written in terms of the vehicle state for the purposes of the analysis. Simulations of the closed-loop dynamics are provided to demonstrate the performance of the proposed algorithm.

The content is organized as follows: Section 2 describes the landing task; the differential equations representing the motion model for small-scale VTOLs and the modelling of the environment. Section 3 proposes a control law for the landing task in obstacle-free environments and provides a detailed stability analysis. Section 4 considers the case where there are obstacles in space and provides an analysis for the proposed control. Section 5 reports on some simulation results for both obstacle-free and cluttered environments. The paper concludes with some final comments in Section 6.

\section{SYSTEM AND ENVIRONMENT MODELLING}

In this section we introduce the model used throughout the document to describe the vehicle dynamics and the environment.

Consider a Vertical-Take-Off-and-Landing (VTOL) UAV vehicle equipped with an Inertial Measurement Unit (IMU) and suitable filtering algorithms to provide reliable estimates of attitude and rotational velocities, as well as a camera, an exteroceptive passive sensor whose output is rich in information. We assume that the vehicle is equipped with vision processing capability to identify a point-feature target and compute optical flow over the full image (Horn and Schunck, 1981).

A complete model of a VTOL aircraft includes gyroscopic and aerodynamics effects, as well as aerodynamic disturbances. In order to simplify the analysis, we neglect all second order terms (Mahony and Hamel, 2004). The neglected aerodynamics forces are dissipative and do not significantly effect the closed-loop response. Rotational dynamics of small-size VTOL vehicles are usually faster than translational ones, leading to a hierarchical control design methodology. The natural time-scale separation between the translational dynamics (slow time-scale) and the orientation dynamics (fast time-scale) allows one to design decoupled position and orientation controllers (Khalil, 2002; Hérissé et al., 2012). The high-gain attitude control effectively dominates the attitude dynamics of the vehicle and allow one to consider a pure translational model

$$
\begin{aligned}
\dot{\xi} & =v \\
m \dot{v} & =m g e_{3}-T R e_{3},
\end{aligned}
$$

in which $\xi$ and $v$ represent the UAV position and velocity respectively, both expressed in an inertial frame with the third direction aligned with the gravitational field, $m$ represents the mass of the VTOL-UAV (supposed constant), $g$ is the magnitude of the gravity force acting on the vehicle and $e_{3}$ is the third canonical basis vector. The variable $T$ represents the thrust magnitude and $R$ is the rotation matrix representing the orientation of the body-fixed frame with respect to the inertial frame. The high-gain attitude control assumption means that the rotation $R$ can be viewed as a control input and we write $u=-T R e_{3}$ to represent the resulting control input to the translational dynamics of the system

$$
\begin{aligned}
\dot{\xi} & =v \\
m \dot{v} & =m g e_{3}+u .
\end{aligned}
$$

In order to develop the dynamics equation of image points, in the following we suppose that the frame representing the position and orientation of the camera in the world frame is rigidly attached to the body-fixed frame, such that the linear transformation relating them degenerates to the identity matrix and their dynamics are the same. Projections of world points on the image surface are points, usually called point features or features, whose dynamics depend on the geometry of the image surface and on the physical parameters of the camera. A common choice in the aerial robotics field is to model the dynamics of the point features by using the spherical projection model for a calibrated camera as shown by Ma et al. (2004). Consider a reference frame attached to the camera. If $\bar{P}$ is a generic point in the 3-D space, we denote with $P$ the vector connecting the centre of the camera reference frame to the point $\bar{P}$ and with $p$ the unit vector representing the direction of $P$, i.e. its projection on a sphere of unit radius, that represents the spherical image surface. The expression of the vector $p$ and its derivative are

$$
p=\frac{P}{|P|}, \quad \dot{p}=-\frac{\pi_{p}}{|P|} v,
$$

where $^{1}$

$$
\pi_{p}=\left(I_{3}-p p^{\top}\right)
$$

is the projector in the space tangent to the spherical image surface at point $p$, having the following properties

$$
\begin{aligned}
p^{\top} \pi_{p} & =\pi_{p} p=0, \\
\pi_{p} \pi_{p} & =\pi_{p} .
\end{aligned}
$$

We will assume that the desired landing point $\bar{P}_{L}$ is contained locally in a planar surface whose normal $\eta$ is known or can be estimated from image features (see, for instance, Ma et al. (2004)). In general, the normal direction cannot be easily determined unless a good knowledge of the environment is available. There are, however, certain special cases where it is relatively straightforward to extract it, such as the situation where the target lies on a flat ground. In this case $\eta$ is obviously the gravitational direction $e_{3}$ and can be directly provided the IMU sensor.

A soft landing manoeuvre consists in progressively reducing the distance between the vehicle and the desired landing point, ensuring it reaches the zero value with zero speed. The environment enforces a physical constraint on

\footnotetext{
1 The symbol $I_{3}$ represents the identity matrix of dimension 3 by 3 .
} 
the vehicle height: it has to be all time strictly positive. Indeed, the desired landing point will always lie on the boundary of the admissible region of the space, an asseveration that requires careful consideration in the stability analysis.

We approximate the cluttered environment by a collection of fixed spherical objects. For a given obstacle, let $\bar{P}_{o}$ denote the closest point on the surface of the obstacle to the camera. Let $P_{o}$ be the vector from camera to $\bar{P}_{o}$ and define $d_{0}=\left|P_{o}\right|$ to be the distance from camera to the obstacle. The vector $p_{o}$ denotes the projection of $P_{o}$ onto the spherical image surface, $p_{o}=P_{o} / d_{o}$. Note that $p_{o}$ is orthogonal to the tangent plane of the spherical obstacle. We assume that the de-rotated optic-flow $\Phi$ is computed at all points in the image. The divergence of the optic flow is a scalar field that can be computed from the optic field using either differentials of the flow or a vector convolution. Locally around $p_{0}$ in the image the divergence of the optical flow is related to the scaled rate of change of distance

do we need some reference here?

$$
\operatorname{div} \Phi\left(p_{o}\right)=-2 \frac{\dot{d}_{o}}{d_{o}} .
$$

In the same manner, denoting the flow over the projection of the ground plane spherical image by $\mathcal{D} \subset S^{2}$ one can show that

$$
\frac{v}{h}=\Lambda \int_{\mathcal{D}} \Phi(p) d p
$$

for a matrix $\Lambda>0$ that can be computed from knowledge of the size of $\mathcal{D}$ (Hérissé et al., 2012) and where $h$ is the distance from the camera to the landing surface plane in the normal direction $\eta$. We will again use the expression $v / h$ in the control analysis rather than writing the dependence on the optic flow field directly. A key aspect of the optic flow measurements is that the resulting measurements always depend inversely on distance to the observed as well as depending linearly on the camera velocity. This singularity in the velocity leads to much of the nice properties of optic flow as a cue for obstacle avoidance, but also causes significant complications in the analysis.

We will assume further that an obstacle-free path to the landing point exists and that the landing point is visible at all times during the evolution of the closed-system. Clearly this assumption will fail in highly cluttered environments and some further hybrid control laws, switching between different target points, or adding feed forward driving terms would need to be considered to overcome this in practice. Considering this issue is beyond the scope of the present paper as we concentrate on the fundamental nonlinear complexity of the problem. For the same reasons, although we recognise that real obstacles are not spherical and a practical implementation of these ideas will need to address these issues, we will not discuss this further in the present paper.

\section{LANDING IN OBSTACLE-FREE ENVIRONMENTS}

In this section, we consider the landing task in an obstaclefree environment. The proposed solution represents a vari- ant of the controllers formerly developed by Hérissé et al. (2010, 2012), that were proportional-integral nonlinear controllers, based on optical-flow data only. Here, we avoid the integration of the always noisy optical-flow measurements, by substituting it by the spherical image point of the landing point. The advantage in avoiding the integral component may become relevant in real applications where the noise in the optical flow measurement is not negligible, e.g. when the limited field of view does not allow to properly average the corrupted measurements, leading to a diverging integral term.

Note that, given any desired landing point, one can use a simple change of coordinates and rewrite the system as if the landing point was the origin of the $3-\mathrm{D}$ space. Then, we assume that the desired landing point is $\bar{P}_{L}=(0,0,0)^{\top}$.

Theorem 1. Consider the system (2) subjected to the control input

$$
u_{\mathrm{L}}=-m g e_{3}-m k\left(\frac{v}{h}+\frac{\xi}{|\xi|}\right)
$$

then, for any initial condition such that $h(0)>0$ and for some $k>0$ the following assertions hold

(1) $h(t)>0, \forall t$ (implying that the dynamics of the closedloop system are well defined $\forall t$ );

(2) $h(t)$ and $\dot{h}(t)$ converge to zero asymptotically;

(3) $v(t)$ converges to zero asymptotically;

(4) $\xi(t)$ converges to zero asymptotically;

(5) the control law (8) is bounded $\forall t$.

The proof of the theorem is given in Appendix A.2.

It is worth noticing that, if we assume that the normal direction to the plane is known, the proposed control input can be computed by using IMU data, Optical Flow measurement and the image of the desired landing point.

\section{LANDING AMONG OBSTACLES}

Navigation and control of vehicles in cluttered environments is a central topic in the area of field robotics. Several solutions have been developed in the literature to cope with this problem. The most familiar approach is certainly the so-called artificial potential fields from Khatib (1986) where the key idea consists in building artificial repulsive force fields that depend linearly on the inverse of the distance to the sensed obstacles. The main problems ${ }^{2}$ of the approach that arise are due to the presence of local minima that can entrap the robot, preventing it from the convergence to the desired position (a problem related to the geometry of the environment and to the incomplete knowledge of it), as discussed by Borenstein and Koren (1989), and to the need of measuring the distance to the obstacles, since the typical sensor suite embarked consists of an inertial measurement unit (IMU), and a camera providing video data.

The key challenge here considered is to slightly modify the control strategy in order to avoid obstacles while performing the landing task using the minimal sensor suite carried by the vehicle. In particular, we exploit optical flow measurement to address the problem of a unified control

2 Details on drawbacks of these techniques and possible solutions have been described by Adeli et al. (2011). 
task incorporating obstacle avoidance.

At first glance, one can exploit the optical-flow measurements for obstacle avoidance purposes by adding to the nominal controller (8) a dissipative term

$$
k_{D} D_{i} p_{i}=k_{D} \frac{\dot{d}_{i}}{d_{i}} p_{i}, \quad d_{i}=\left|P_{i}\right|
$$

for each obstacle, where $D_{i}=\dot{d}_{i} / d_{i}$ is proportional to the measured optical-flow divergence (7). Those terms act along the directions $p_{i}$ of the obstacles, and adding them to the landing control input one gets

$$
u=u_{L}+u_{D}, \quad \text { where } u_{D}=k_{D} \sum_{i=1}^{n} D_{i} p_{i}, K_{D}>0 \text {. }
$$

Since the derivative of the distance, i.e. $\dot{d}_{i}=-v^{\top} p_{i}$, is the projection of the velocity vector along the direction $p_{i}$, it is negative when the vehicle approaches the obstacle. The following storage function (also exploited in the proof of Th. 1, see eq. (A.8)),

$$
\mathcal{L}=k|\xi|+\frac{|v|^{2}}{2}
$$

shows, via its time derivative

$$
\dot{\mathcal{L}}=-k \frac{|v|^{2}}{h}-k_{D} \sum_{i=1}^{N} \frac{\left|p_{i}^{\top} v\right|^{2}}{d} \leq 0
$$

that the control input $u_{D}$ acts as a dissipative term, whose intensity can be modulated by means of the gain $k_{D}$. The vehicle will slow down in the unwanted direction, while preserving its motion in the rest of the space. Being $u_{D}$ the sum of pure dissipative terms, it is hard to ensure by means of the control input (9) that there is no contact with the obstacles. To overcome this issue, one can suggest the design of a repulsive field around each obstacle $i$, derived from the following function:

$$
\phi_{i}\left(d_{i}\right)=\left\{\begin{array}{cc}
k_{R}\left(d_{i}(t)\left(\gamma_{i}(t)-1\right)+d_{i}(0)\right), & d_{i}<d_{i}(0) \\
0, & d_{i} \geq d_{i}(0)
\end{array}\right.
$$

where $k_{R}$ is a small positive gain $\left(k_{R} \ll k\right)$ and $\gamma_{i}$ is given by an integral of the term $D_{i}$

$$
\gamma_{i}(t)=\int_{0}^{\tau} \frac{\dot{d}_{i}(\tau)}{d_{i}(\tau)} d \tau=\ln \left(\frac{d_{i}(t)}{d_{i}(0)}\right)
$$

Choosing the following expression of the repulsive force:

$$
F_{i}\left(d_{i}\right)=k_{R} \gamma_{i} p_{i}
$$

It follows that near the obstacle the term $\gamma_{i}$ is negative and hence the force (even small) is actually driving the vehicle away from the obstacle neighborhood. For $n$ visible obstacles in the environment, we design the obstacleavoidance control input by summing all the contributions, generated by using eq. (12) for each obstacle:

$$
u_{R}=k_{R} \sum_{i=1}^{n} \gamma_{i} p_{i}
$$

By defining the control action $u$ as $u=u_{L}+u_{D}+u_{R}$ we get the following result:

Theorem 2. Consider the system (2) under the control input

$$
u=u_{L}+u_{D}+u_{R}
$$

Assume the environment is populated by $n(n \geq 1)$ spherical obstacles and that the desired point is reachable

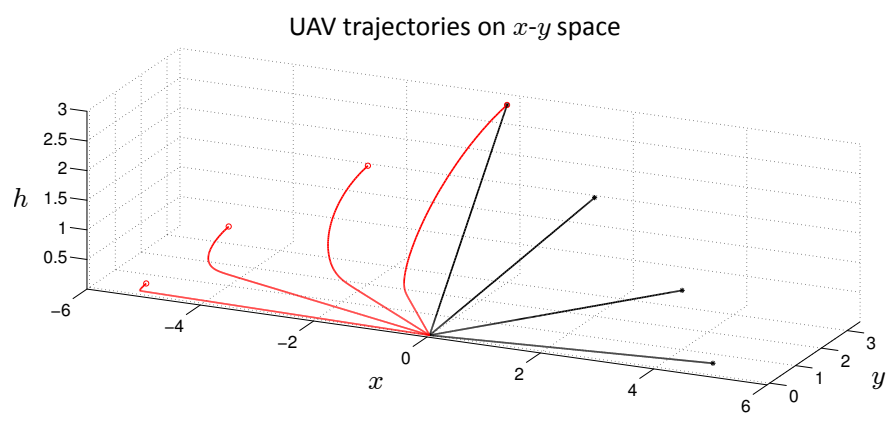

Fig. 1. Trajectories in the 3-D space $(x-y$ - $h)$ for different initial conditions. Black lines correspond to zero initial velocities. Red lines correspond to initial velocity $V(0)=(-1,0,-1)^{\top}$.

from any initial point $\xi(0)$ in the admissible space. Assume there is no contact between the obstacles and the landing plane and choose $k_{R}$ of (13) small with respect to $k$ enough. Then, for any initial conditions such that $h(0)>0$

(1) All solutions converge to $E_{a} \cup E_{u}$. With $E_{a}=(0,0)$ and

$$
E_{u}=\left\{\left(\xi^{*}, 0\right) \text { s.t. } k \frac{\xi^{*}}{\left|\xi^{*}\right|}=k_{R} \sum_{i=1}^{n} p_{i}\left(\xi^{*}\right) \gamma_{i}\left(\xi^{*}\right)\right\}
$$

(2) The point $E_{a}$ is a locally attractive fixed point;

(3) Any point of $E_{u}$ is an unstable equilibrium. More precisely, for any $\left(\xi^{*}, 0\right) \in E_{u}$ and for any neighbourhood $\mathcal{U}$ of $\left(\xi^{*}, 0\right)$, there exists $(\tilde{\xi}, \tilde{V}) \in \mathcal{U}$ such that the trajectories of the system starting from $(\tilde{\xi}, \tilde{V})$ converges to $E_{a}$.

The proof is reported in Appendix A.3.

\section{SIMULATION RESULTS}

Here we show some simulation results obtained using the landing controller described in sec. 3, and its extension for the cluttered environment case, as described in sec. 4 . All simulations are conducted including the rotational dynamics of the vehicle as well as some additive noise in the measurements of the optical flow and the bearing direction of the obstacles. To show the behaviour of the system under the control law (8), we chose a set a of different initial conditions both for position and velocity vectors.

Fig. 1 represents trajectories in the $3-\mathrm{D}$ space resulting from those initial conditions. The environment is symmetric and we chose to confine the evolutions of the system in the subspace $y>0$. Moreover, we chose initial conditions that are symmetric with respect to the $y$ - $h$ plane to visually separate trajectories resulting from different initial velocities. Indeed, initial conditions with $x(0)>0$ correspond to zero initial speed and their evolutions are drawn in black (starting position are marked with a star symbol). As might be expected from the symmetry of the system, the UAV is driven along straight lines directly to the landing point.

On the other hand, red lines (and their relative starting points, marked with red circles) represent the evolutions of the system corresponding to $v(0)=(-1,0,1)^{\top}$, i.e. when the initial conditions are such to drive the vehicle away 


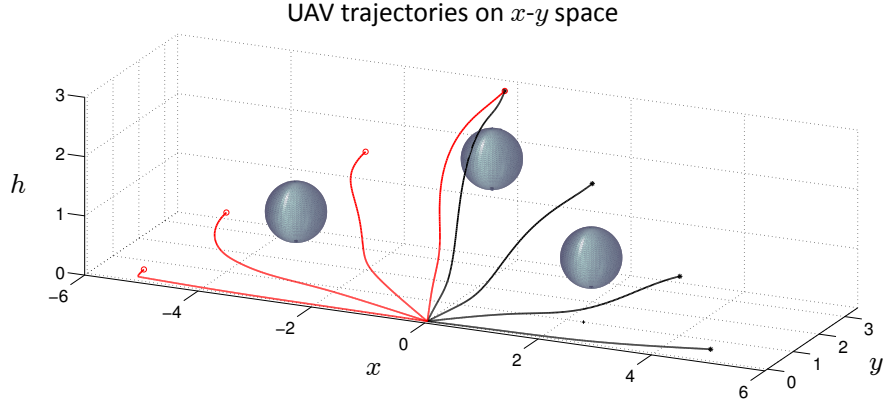

Fig. 2. Trajectories in the 3-D space $(x-y-h)$ for different initial conditions, case with multiple obstacles. Black lines correspond to zero initial velocities. Red lines correspond to initial velocity $V(0)=(-1,0,-1)^{\top}$.

from the landing point and towards the ground plane. Consider the trajectory resulting from initial condition $\xi(0)=(-5,0,-0.2)^{\top}$ (leftmost in Fig. 1). As $h$ approaches zero, the control input grows and compensates the initial speed. The vertical speed approaches zero and the vehicle slowly slides in the direction of the landing point. The system shows an analogous behaviour when initial conditions are such that the initial height is close to zero, as can be seen by looking at the trajectory starting from $\xi(0)=(5,0,0.2)^{\top}$ (rightmost in Fig. 1). In those cases, the convergence to the landing point may require a big amount of time and the small value of the height may prevent the use of the proposed control in real systems.

The addition of the repulsive action and damping terms to the control input, (recall eq. (14)), modifies all the trajectories. Simulations results for this case are shown in Fig. 2. The initial conditions are the same as the previous case. The control strategy is in some sense conservative, since it modifies even those trajectories which would not collide with the obstacles in any case. This fact can be easily noticed by comparing Fig. 1 and Fig. 2.

\section{CONCLUSIONS AND FUTURE WORKS}

In this paper we addressed the problem of landing a VTOL-UAV in obstacle-free or cluttered environments. The proposed control law exploits measurements given by a camera attached to the vehicle as well as an IMU, a common sensor suite for an unmanned aerial vehicle. The simulation results shows the effectiveness of the control law in all the environments. Here we considered spherical objects, placed at a non-zero height from the ground. Considering non-spherical objects involves the estimate of the normal to their surface, and would be a useful extension towards a real-world implementation of the algorithm. We also plan to prosecute the work towards the implementation on a real small-scale UAV.

\section{REFERENCES}

Adeli, H., Tabrizi, M.H.N., Mazloomian, A., Hajipour, E., and Jahed, M. (2011). Path planning for mobile robots using iterative artificial potential field method. International Journal of Computer Science (IJCSI), 8(2), 28-32.

Andert, F., Adolf, F., Goormann, L., and Dittrich, J. (2011). Mapping and path planning in complex envi- ronments: An obstacle avoidance approach for an unmanned helicopte. In Proceeding of IEEE International Conference on Robotics and Automation, 745-750.

Beyeler, A., Zufferey, J.C., and Floreano, D. (2009). Vision-based control of near-obstacle flight. Journal of Autonomous Robots, 201-219.

Blösch, M., Weiss, S., Scaramuzza, D., and Siegwart, R. (2010). Vision based mav navigation in unknown and unstructured environments. In Proceedings of IEEE International Conference on Robotics and Automation, 21-28.

Borenstein, J. and Koren, Y. (1989). Real-time obstacle avoidance for fast mobile robots. IEEE Transactions on Systems, Man and Cybernetics, 19(5), 1179-1187.

Chahl, J.S., Srinivasan, M.V., and Zhang, S.W. (2004). Landing strategies in honeybees and applications to uninhabited airborne vehicles. International Journal of Robotics Research, 101-110.

Courbon, J., Mezouar, Y., Guenard, N., and Martinet, P. (2010). Vision-based navigation of unmanned aerial vehicles. IEEE Transactions on aerospace and electronic systems, 18, 789-7997.

Davison, A.J., Molton, I.D.R.N.D., and Stasse, O. (2011). Monoslam: Real-time single camera slam. IEEE Transactions on pattern analysis and machine intelligence, 29(6), 1052-1067.

Flores, M.E. and Milam, M.B. (2006). Trajectory generation for differentially flat systems via nurbs basis functions with obstacle avoidance. In Proceeding of American Control Conference (ACC), 5769-5775.

Geyer, M.S. and Johnson, E.N. (2006). 3d obstacle avoidance in adversarial environments for unmanned aerial vehicles. In AIAA Guidance, Navigation, and Control Conference and Exhibit.

Green, W.E. and Oh, P.Y. (2008). Optic flow based collision avoidance. IEEE Robotics $\&$ Automation Magazine, 96-103.

Hérissé, B., Hamel, T., Mahony, R., and Russotto, F.X. (2010). The landing problem of a vtol unmanned aerial vehicle on a moving platform using optical flow. In IEEE/RSJ International Conference on Intelligent Robots and Systems (IROS), 1600-1605.

Hérissé, B., Hamel, T., Mahony, R., and Russotto, F.X. (2012). Landing a vtol unmanned aerial vehicle on a moving platform using optical flow. IEEE Transaction on robotics, 28(1), 77-89.

Hérissé, B., Russotto, F.X., Hamel, T., and Mahony, R. (2008). Hovering flight and vertical landing control of a vtol unmanned aerial vehicle using optical flow. In IEEE/RSJ International Conference on Intelligent Robots and Systems (IROS), 801-806.

Horn, B.K.P. and Schunck, B.G. (1981). Determining optical flow. Artificial Intelligence, 17, 185-203.

Humbert, J.S., Murray, R.M., and Dickinson, M.H. (2005). Pitch altitude control and terrain following based on bioinspired visuomotor convergence. In AIAA Conference on Guidance, Navigation and Control.

Kaiser, M.K., Gans, N.R., , and Dixon, W.E. (2010). Vision-based estimation for guidance, navigation, and control of an aerial vehicle. IEEE Transactions on aerospace and electronic systems, 46(3), 1064-1077.

Khalil, H.K. (2002). Nonlinear Systems. Prentice-Hall. 
Khatib, O. (1986). Real-time obstacle avoidance for manipulators and mobile robotics. The International Journal of Robotics Research, 5(1), 90-98.

Koenderink, J. and van Doorn, A. (1987). Facts on optic flow. Journal of Biological Cybernetics.

Lee, D., Ryan, T., and Kim, H.J. (2012). Autonomous landing of a vtol uav on a moving platform using imagebased visual servoing. In IEEE International Conference on Robotics and Automation, 971-976.

Ma, Y., Soatto, S., Košeckà, J., and Sastry, S.S. (2004). An Invitation to 3-D vision. Springer.

Mahony, R. and Hamel, T. (2004). Robust trajectory tracking for a scale model autonomous helicopter. International Journal of Robust and Nonlinear Control, 14(12).

Marconi, L., Isidori, A., and Serrani, A. (2002). Autonomous vertical landing on an oscillating platform: an internal-model based approach. Automatica, 38, 21-32.

McCarthy, C., Barnes, N., and Mahony, R. (2012). A unified strategy for for landing and docking using spherical flow field divergence. IEEE Transactions on Pattern Analysis and Machine Intelligence, 34(5), 1024-1031.

Meister, O., Frietsch, N., Ascher, C., and Trommer, G.F. (2009). Adaptive path planning for vtol-uavs. Aerospace and Electronic Systems Magazine, IEEE, 24(7), 36-41.

Michael, N., Shen, S., Mohta, K., Mulgaonkar, Y., Kumar, V., Nagatani, K., Okada, Y., Kiribayashi, S., Otake, K., Yoshida, K., Ohno, K., Takeuchi, E., and Tadokoro, S. (2012). Collaborative mapping of an earthquakedamaged building via ground and aerial robots. Journal of Biological Cybernetics, 29, 832-841.

Milford, M.J., Schill, F., Corke, P., Mahony, R., and Wyeth, G. (2011). Aerial slam with a single camera using visual expectation. In Proceedings of IEEE International Conference on Robotics and Automation, 25062512.

Ruffier, F. and Franceschini, N. (2004). Visually guided micro-aerial vehicle: automatic take off, terrain following, landing and wind reaction. In Proceedings of IEEE International Conference on Robotics and Automation.

Ruffier, F. and Franceschini, N. (2005). Optic flow regulation: the key to aircraft automatic guidance. Journal of Robotics and Automation Systems, 177-194.

Srinivasan, M., Zhang, S., Chahl, J.S., Barth, E., and Venkatesh, S. (2000). How honeybees make grazing landings on flat surfaces. Journal of Biological Cybernetics.

\section{Appendix A}

\section{A.1 Preliminary technical lemmas}

All time-dependent functions involved in the statements of these lemmas are assumed to be defined on $[0,+\infty)$ and continuous. The state variable $x$ belongs to $\mathbb{R}^{n}$, with $n$ a positive integer. Also, o $t$ ) denotes any bounded vectorvalued function whose norm tends to zero when $t$ tends to infinity.

Lemma 3. Consider the system

$$
\dot{x}=-k(t) x+p(t) \text {. }
$$

If $k(t)$ is positive $(\forall t)$ and tends to infinity, and if $|p(t)|$ is bounded, then (any solution) $x(t)$ (to this system) converges to zero.
Proof. The lemma's assessment is obvious after observing that, by replacing the time index $t$ by the new time-scale index $s(t):=\int_{0}^{t} k(\tau) d \tau$ (note that $s$ tends to infinity if and only if $t$ also tends to infinity), the considered system rewrites as

$$
\frac{d}{d s} x=-x+o(s)
$$

with $o(s):=\frac{p(t)}{k(t)}$. This is a stable linear system perturbed by a vanishing additive perturbation.

Lemma 4. Consider the system

$$
\dot{x}=-k(t)((1+o(t)) x+p(t)) \text {. }
$$

If $k(t)$ is positive $(\forall t)$ and tends to infinity, and if $|p(t)|$ is bounded, then $|x(t)|$ is bounded.

Proof. Again the proof of this lemma is quite simple after using the same change of time-scale index as for the previous lemma. This yields

$$
\frac{d}{d s}=-(1+\bar{o}(s)) x-\bar{p}(s),
$$

with $\bar{o}(s)=o(t)$ and $\bar{p}(s)=p(t)$. Since $(1+\bar{o}(s))$ tends to 1 , this is basically a linear system perturbed by a bounded additive perturbation.

Lemma 5. Consider the system

$$
\dot{x}=-k(t)((1+o(t)) x+p(t)),
$$

If $k(t)$ is positive $(\forall t)$ and tends to infinity, and if $|p(t)|$, $|\dot{p}(t)|$, and $k(t) o(t)$ are bounded. Then, $(x(t)+p(t))$ converges to zero as $t$ goes to infinity.

Proof. Define $y:=x+p$, then

$$
\begin{aligned}
\dot{y} & =-k(t)([1+o(t)] x+p)+\dot{p} \\
& =-k(t)[1+o(t)] y+k(t) o(t) p+\dot{p} .
\end{aligned}
$$

and a direct application of Lemma 3 yields the desired result.

Lemma 6. Consider two positive functions $\alpha(t)$ and $\beta(t)$ such that

$$
\lim _{t \rightarrow \infty} \int_{0}^{t} \alpha(s) d s=+\infty, \quad \lim _{t \rightarrow \infty} \beta(t)=1,
$$

then

$$
\lim _{t \rightarrow \infty} \int_{0}^{t} \alpha(s) \beta(s) d s=+\infty .
$$

Proof. There exists a time $t^{*}$ such that $\beta(t)>\beta_{0}>$ $0, \forall t>t^{*}$. Therefore, when $t>t^{*}$ one has

$$
\int_{0}^{t} \alpha(s) \beta(s) d s>\int_{0}^{t^{*}} \alpha(s) \beta(s) d s+\beta_{0} \int_{t^{*}}^{t} \alpha(s) d s,
$$

with the last term in the right-hand side of the inequality converging, by assumption, to infinity.

\section{A.2 Proof of Theorem 1}

Given the control (8) applied to the system (2), the equation of the closed-loop system is

$$
\ddot{\xi}=-k\left(\frac{\dot{\xi}}{h}+\frac{\xi}{|\xi|}\right)=-k\left(\frac{\dot{\xi}}{h}+\alpha \frac{\xi}{h}\right),
$$


with

$$
\alpha(t)=\frac{h(t)}{|\xi(t)|}, \quad \alpha(t) \in(0,1], \forall t .
$$

Proof of item 1: The vehicle is initially above the target, i.e. $h(0)>0$. Given an (any) initial condition $(\xi(0), v(0))$, as long as $h(t)$ does not reach zero, the solution to the above equation is well defined and unique. Let us first show that $h(t)$ cannot reach zero in finite time. By definition $h$ is the third component of $\xi$. Therefore, in view of (A.4), the time-evolution of $h$ is given by

$$
\ddot{h}=-k\left(\frac{\dot{h}}{h}+\alpha\right) \text {. }
$$

Integration of both sides of this equality yields

$$
\dot{h}(t)=\dot{h}(0)-k \ln \left(\frac{h(t)}{h(0)}\right)-k \int_{0}^{t} \alpha(s) d s .
$$

This relation is valid as long as $h(t) \neq 0$. Now, consider the following positive function

$$
\mathcal{L}=k|\xi|+\frac{|v|^{2}}{2}
$$

whose time-derivative along a solution to System (A.4) is

$$
\dot{\mathcal{L}}=-k \frac{|v|^{2}}{h} \leq 0 \text {. }
$$

One deduces from the previous two relations that $|\xi|(h)$ and $|v|(\geq|\dot{h}|)$ are uniformly bounded with respect to the initial condition, as long as the solution to the system is defined, i.e. as long as $h$ remains positive. If we assume that $h$ reaches zero at the finite time-instant $T_{s}>0$, then the term $\ln \left(\frac{h(t)}{h(0)}\right)$ appearing in the equality (A.7) tends to infinity when $t$ tends to $T_{s}$, whereas all other terms involved in this equality remain bounded. This assumption thus yields a contradiction. Therefore $h$ is never equal to zero, and the solution to System (A.4) is well defined and unique for $t \in[0, \infty)$.

Proof of item 2: Using the following change of variables

$$
z(t)=h(t) \exp \left(\frac{\dot{h}}{k}\right)
$$

one gets $\dot{z}(t)=-\alpha(t) z(t)$, a first order differential equation whose solution is

$$
z(t)=z(0) \exp \left(-\int_{0}^{t} \alpha(\tau) d \tau\right) .
$$

In order to study the evolution of $(h, \dot{h})$, we can consider two different cases, depending on the sign of the initial vertical velocity.

Case 1: $\dot{h}(0)<0$. Let us show that $\dot{h}(t)<0, \forall t$.

We make a proof by contradiction and assume that there exists $t^{*}$ such that $\dot{h}\left(t^{*}\right)=0$ and $\ddot{h}\left(t^{*}\right) \geq 0$. Then, according to (A.6), $\ddot{h}\left(t^{*}\right)<0$. A clear contradiction.

Case 2: $\dot{h}(0) \geq 0$. If $\dot{h}(t)$ were always positive, or equal to zero, then, according to (A.10), $z(t)$ would be nondecreasing. This contradicts relation (A.11) which implies that $z(t)$ is strictly decreasing. Therefore there exists a time instant $T$ such that $\dot{h}(T)<0$, and we are brought back to Case 1 with $T$ taken as the new origin of time.

By considering these two cases, we have shown that $\dot{h}(t)$ is strictly negative after a finite time. Let us now show that $h(t)$ and $\dot{h}(t)$ converge to zero. Since $h(t)$ is positive and decreases after a finite time-instant, it converges to some limit $h_{m}$ which is either positive or equal to zero. Recall that the boundedness of $\xi$ has already been proven so that $\xi_{M}:=\sup _{t \in[0,+\infty)}|\xi(t)|$ is a positive finite number. Let us assume that $h_{m}$ is positive, then $\alpha(t)>\frac{h_{m}}{\xi_{M}}=\alpha_{m}>0$, $\forall t$. Relation (A.11) then implies

$$
z(0) e^{-\alpha_{m} t}>z(t) \geq z(0) e^{-t},
$$

and thus the convergence of $z(t)$ to zero. This in turn implies the convergence of $h(t)$ to zero, since $|\dot{h}(t)|(\leq$ $|v(t)|)$ is bounded. From this contradiction, one deduces that $h_{m}=0$ and thus that $h(t)$ converges to zero.

Finally, using the fact that $k \alpha(t)$ is bounded and that $k / h(t)$ tends to infinity, the application of Lemma 3 to the equation (A.6) establishes the convergence of $\dot{h}(t)$ to zero.

Proof of item 3: We first show that the ratio $\frac{|v|}{h}$ is bounded. To this aim let us consider the equation governing the evolution of this ratio, as deduced from (A.4)

$$
\frac{d}{d t}\left(\frac{|v|}{h}\right)=-\frac{k}{h}\left(\left[1+\frac{\dot{h}}{k}\right] \frac{|v|}{h}+\cos (\beta)\right) .
$$

with $\beta$ the angle between $v$ and $\xi$, so that $\cos (\beta)=\frac{v^{T} \xi}{|v||\xi|}$. Using the fact that $k / h$ tends to infinity and that $\dot{h} / k$ tends to zero, the boundedness of $\frac{|v|}{h}$ is simply obtained by applying Lemma 4 to this equation. From there, the convergence of $v$ to zero just follows from the convergence, previously proven, of $h$ to zero.

Proof of item 4: Since

$$
\frac{d}{d t}\left(\frac{\xi}{|\xi|}\right)=\left(I-\frac{\xi \xi^{\top}}{|\xi|^{2}}\right) \frac{v}{|\xi|},
$$

and since $\frac{|v|}{|\xi|}\left(\leq \frac{|v|}{h}\right)$ tends to zero, the time-derivative of $\frac{\xi}{|\xi|}$ tends to zero, and is thus bounded. Consider now the dynamics of the ratio between $\dot{\xi}(t)$ and $h(t)$

$$
\frac{d}{d t}\left(\frac{\dot{\xi}}{h}\right)=-\frac{k}{h}\left(\left[1+\frac{\dot{h}}{k}\right] \frac{\dot{\xi}}{h}+\frac{\xi}{|\xi|}\right),
$$

The application of Lemma 5 to this equation yields

$$
\left(\frac{\dot{\xi}}{h}+\frac{\xi}{|\xi|}\right)(t)=o(t) \text {. }
$$

Pre-multiplying both members of the above equality by $h \xi^{\top}$, one obtains the equation

$$
\frac{d}{d t}|\xi|^{2}+\bar{\alpha}(t)|\xi|^{2}=0
$$

with

$$
\bar{\alpha}(t):=2 \alpha(t)\left(1-\frac{\xi(t)^{\top}}{|\xi(t)|} o(t)\right) .
$$

whose solution must satisfy 


$$
|\xi(t)|^{2}=|\xi(0)|^{2} \exp \left(-\int_{0}^{t} \bar{\alpha}(s) d s\right) .
$$

Since $h$ and $\dot{h}$ converge to zero, one deduces from (A.7) that the integral of $\alpha$ tends to $+\infty$. Therefore, by application of Lemma 6 , the integral of $\bar{\alpha}$ also tends to $+\infty$. The convergence of $\xi$ to zero then follows from the previous equality.

Proof of item 5: The boundedness of the control $u_{L}$ given by (8) simply follows from the previously proven boundedness of $v / h$.

\section{A.3 Proof of Theorem 2}

Proof of item 1: Consider the following Lyapunov function:

$$
\mathcal{L}=k|\xi|+\frac{1}{2}|v|^{2}+\sum_{i=1}^{n} \phi_{i},
$$

whose derivative is

$$
\dot{\mathcal{L}}=-\frac{|v|^{2}}{2}-k_{D} \sum_{i=1}^{n} \frac{\dot{d}_{i}^{2}}{d_{i}} .
$$

Let's divide the analysis in two cases, related to the behaviour along the vertical axis and let define $h_{\epsilon}$, the value of $h$ that characterises the height of the nearest obstacle to the ground.

Case 1: $h \geq h_{\epsilon}, \forall t$. Then $\dot{\mathcal{L}}=0$ implies that the velocity vector is null, and the equilibrium $(\xi, v)=\left(\xi^{*}, 0\right) \neq(0,0)$ is a solution of the implicit equation $\ddot{\xi}^{*}=0$. This implies

$$
k \frac{\xi^{*}}{\left|\xi^{*}\right|}=k_{R} \sum_{i=1}^{n} \gamma_{i} p_{i} .
$$

Case 2: $h<h_{\epsilon}$. In the hypothesis that the minimum height of the obstacles is such that their residual influence on the vehicle dynamics is negligible, then $(\xi, 0)$ is converging to the landing point $(0,0)$. This case is similar to consider the neighbourhood of the landing point itself, then one can rely on the same arguments used for the next item.

Proof of item 2: To show that $E_{a}$ is a locally attractive point, consider the dynamics of the system in the neighborhood of the landing point i.e. when $h<h_{\epsilon}$. Recalling eq. (10), one knows that the terms $\gamma_{i}$ are negative or null. By neglecting the effect of the dissipative term due to $u_{D}$ it is straightforward to verify that the inner product $\eta^{\top} p_{i}>0$ and hence the projected dynamics are approximately

$$
\ddot{h}=-k\left(\frac{\dot{h}}{h}+\alpha^{\prime}\right), \text { where } \alpha^{\prime}=\frac{h}{|\xi|}+\frac{k_{R}}{k} \sum_{i=1}^{n} \eta^{\top} p_{i}\left|\gamma_{i}\right|
$$

Thus the same arguments used in the proof of Th. 1 apply and one can conclude that $h$ converges to zero.

Proof of item 3: To show the instability of the set $E_{u}$ we compute the linearization of the error dynamics around the point $\left(\xi^{*}, 0\right) \in E_{u}$.

Let $\chi=(\xi, \dot{\xi})^{\top}$, and recall that in this case, the full system dynamics are

$$
\ddot{\xi}=-k\left(\frac{\dot{\xi}}{h}+\frac{\xi}{|\xi|}\right)+k_{D} \sum_{i=1}^{N} D_{i} p_{i}+k_{R} \sum_{i=1}^{N} \gamma_{i} p_{i} .
$$

Then, by computing the partial derivatives of $\dot{\xi}, \ddot{\xi}$ with respect to the components of the state vector $\chi$, and by

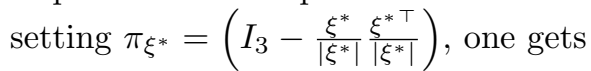

$\dot{\chi}=\left(\begin{array}{c|c}0_{3} & I_{3} \\ \hline-k \frac{\pi_{\xi^{*}}}{|\xi *|}-\sum_{i=1}^{n} \mu_{i}\left(\frac{\pi_{p_{i}} \gamma_{i}}{d_{i}}+\frac{p_{i} p_{i}^{\top}}{d_{i}}\right) & -\frac{I_{3}}{h}-k_{o} \sum_{i=1}^{n} \frac{p_{i} p_{i}^{\top}}{d_{i}}\end{array}\right) \chi$.

Recalling eq. (A.18), and taking the norm of the equilibrium point, it follows that:

$$
\left|\sum_{i=1}^{n} \gamma_{i} p_{i}\right|=\frac{k}{k_{R}}
$$

By choosing $k_{R}$ small enough, it is straight forward to verify that there exists an obstacle $j \in\{1, \ldots, n\}$ such that:

$$
\lim _{k_{R} \rightarrow 0}\left|\sum_{i=1}^{n} \gamma_{i} p_{i}\right|=\left|\gamma_{j} p_{j}\right|=+\infty
$$

Since the obstacles are assumed to be scattered in the environment, the above limit is approximately valid even if $k_{R}$ is only chosen small enough with respect to $k$ and consequently the problem considered is similar to the issue when only a single obstacle is present in the environment. Without loss of generality, define $p=p_{j}, \gamma=\gamma_{j}$, and $d=d_{j}$ and examine the linearization of the system dynamics.

Since the second element of the second row of the matrix above is negative, to prove the instability of the system in the set $E_{u}$, consider the first element of the second row

$$
-k \frac{\pi_{\xi^{*}}}{\left|\xi^{*}\right|}-k_{R}\left(\frac{\pi_{p} \gamma}{d}+\frac{p p^{\top}}{d}\right) .
$$

Note that $\frac{\xi^{*}}{\left|\xi^{*}\right|}= \pm p$, according to the sign of $\gamma$ (recall eq. (A.18)), and consider a rotation matrix $Q$, such that $p=Q e_{3}$ one gets

$$
-\left(\frac{k}{\left|\xi^{*}\right|}+\frac{k_{R} \gamma}{d}\right) \pi_{e_{3}}-\frac{k_{R}}{d} e_{3} e_{3}^{\top} .
$$

If the vehicle is approaching the obstacle, $\gamma$ is negative and from (A.18), it results $k_{R} \gamma=-k$, then one gets

$$
-k\left(\frac{1}{\left|\xi^{*}\right|}-\frac{1}{d}\right) \pi_{e_{3}}-\frac{k_{R}}{d} e_{3} e_{3}^{\top}
$$

whose eigenvalues are

$$
\left\{-k\left(\frac{1}{\left|\xi^{*}\right|}-\frac{1}{d}\right),-k\left(\frac{1}{\left|\xi^{*}\right|}-\frac{1}{d}\right),-\frac{k_{R}}{d}\right\} .
$$

The first two of them are positive in the neighborhood of the obstacle, since $d<\left|\xi^{*}\right|$, thus the matrix is unstable. 